\title{
Shades of Grey
}

Granularity, Pragmatics, and Non-Causal Explanation

\section{Hugh Desmond}

\author{
KU Leuven
}

\begin{abstract}
Implicit contextual factors mean that the boundary between causal and noncausal explanation is not as neat as one might hope: as the phenomenon to be explained is given descriptions with varying degrees of granularity, the nature of the favored explanation alternates between causal and non-causal. While it is not surprising that different descriptions of the same phenomenon should favor different explanations, it is puzzling why redescribing the phenomenon should make any difference for the causal nature of the favored explanation. I argue that this is a problem for the ontic framework of causal and non-causal explanation, and instead propose a pragmatic modal account of causal and non-causal explanation. This account has the added advantage of dissolving several important disagreements concerning the status of non-causal explanation.
\end{abstract}

KEYWORDS: non-causal explanation; modal strength; grain of analysis; information; pragmatics 


\section{INTRODUCTION}

Two obstacles seem to preclude any agreement on how causal explanations should be delimited from non-causal explanations. The first concerns the very definition of causal explanation, which determines how the boundary between causal and non-causal explanation is drawn. Even though most adhere to a relatively narrow definition of causal explanation and thus allow for non-causal explanations (Woodward 2003; Craver 2014), it remains possible to adopt a very wide definition of causal explanation and thus to argue that all purported examples of non-causal scientific explanation are in fact causal (e.g. Skow 2014).

The second obstacle, relatively overlooked by comparison, concerns the pragmatics of causal and non-causal explanations. In a perceptive paper Andersen (Andersen 2016) points out that judging an explanation to be causal seems to be very sensitive to the way in which the explanandum is described. If one varies the grain with which an explanandum is described, previously paradigmatic examples of non-causal explanation no longer seem unproblematically non-causal.

Few if any philosophers would entirely deny the importance of pragmatics. The importance of implicit contextual factors for the analysis of scientific explanations has long been recognized (going back to e.g. Hempel 1942, or Hempel and Oppenheim 1948). However, a separate question arises as to why fine-graining an explanandum should have any effect on our assessment of the causal nature of an explanation - and how precisely this occurs.

If one adopts a purely ontic view of explanation, this may seem like a nonquestion. According to the ontic view, an explanans explains in virtue of picking 
out objective causal (or non-causal, as the case might be) dependencies (Salmon 1989; Woodward 2003; Strevens 2008; Craver 2014). Thus fine-graining an explanandum is simply describing the corresponding phenomenon with increased precision, which in turn helps prevent confusion as to what explanatory dependencies are being picked out. Redescribing examples of causal/non-causal explanation leads to different assessments of their causal nature, simply because different causal/non-causal dependencies are being picked out.

Yet, lurking beneath the surface are regularities that such an ontic view is oblivious to. Our judgment of the causal nature of explanation is very sensitive to slight redescriptions of the phenomenon - as Andersen notes, "slight reformulations of the explanandum will change the relevant explanation(s) from causal to non-causal and back again." (Andersen 2016,2). The problem I wish to draw attention to is why this should be the case at all. After all, strictly speaking, a redescription of a given explanandum actually involves considering a different explanandum, and this explanandum may in turn have many potential explanations. Some of these will pick out causal dependencies, and others non-causal dependencies; some of these explanations will be good, and others bad. Let us assume going forward that there is always a single 'best' explanation (this in fact tends to be assumed in the examples discussed in the literature), so that a single explanans can be associated with a given explanandum. This (best) explanation can be called the associated explanation of given explanandum. Why should redescribing an explanandum affect the causal nature of the associated explanation at all? Why should, for instance, redescribing the bridges of Königsberg lead

\footnotetext{
${ }^{1}$ Just to emphasize, speaking of a redescription (and in a more technical sense: a fine-graining)
} 
to different evaluations of the causal nature of the associated explanation? Why could not redescription only reveal a host of causal explanations, or only a host of non-causal explanations?

If one were to compare a scientific explanation to an object in an old-fashioned, grey-scale photograph (as in Andersen 2016), the redescription is like a grainfocuser that reveals the grey object to be composed of some slightly darker grains (e.g., non-causal explanations), and slightly brighter grains (causal explanations). However, why should this be the case? Why should zooming in not reveal just shades of the same monotone grey? Or why could a scientific explanation not be like a digitized photograph, where zooming in on a grey-scale object reveals a host of perfectly white and perfectly black pixels? From a purely ontic view of explanation, this complex interplay between granularity and our judgments of the causal nature of explanation is more or less a mystery.

In this paper I will propose an account of how the granularity with which explanandum and explanans are described affects our judgments of the causal nature of the associated explanation. I call this the 'pragmatic-modal' view of the causal nature of explanation: it accounts for the relation between granularity and (the judgment of) the causal nature of explanation in terms of how often implicit contextual factors (the 'pragmatic') affect the modal structure of the relation between explanans and explanandum.

After considering some examples in the first section, in the second I define of an explanandum as an operation that 'affects' or 'changes' the causal nature of the associated explanation is a shorthand for the precise but convoluted description of the connection between redescription and the causal nature of an explanation above. 
fine-graining as a set-theoretic operation involving the introduction of additional contrast classes (in continuity with, e.g., Van Fraassen 1980 or Hitchcock 1996). In the third section I draw on Lange's concept of modal strength (but bracket Lange's metaphysics: see Lange 2009) to show how the structure of the relation between explanans and explanandum can be connected with judgments of the explanation as causal or non-causal. In the fourth and final section I bring the two preceding sections together, and account for the fundamental connection between fine- and coarse-graining and the causal nature of explanation. I further illustrate where this approach can be philosophically fruitful: dissolving disagreements concerning the causal nature of paradigmatic cases (Euler's bridges, marbles in bowls, etc.), and dissolving (at least some) disagreements concerning the definition of causal explanation.

\section{The Role of Pragmatics: Some Observations}

Does a mathematical model, such as the Lotka-Volterra model, constitute a causal or non-causal explanation? This obviously depends on how it is used, and in particular, on what explanandum is targeted. For instance, if the explanandum is a general feature of population dynamics, for instance the simple fact of periodicity, then the Lotka-Volterra equations can be used to mathematically deduce periodicity. However, if more specific outcome states are taken as the explanandum, such as the frequencies of hare and lynx in an actual population as they change over time, then additional causal factors will likely have to be invoked. For instance: there may be other marginal predators in the ecosystem preying on the hare; the hare may have other competitors in its habitat; the lynx may be suffer- 
ing from a parasite which prevents the lynx population from reaching certain a density. In this case the Lotka-Volterra model is used as an abstract causal model that represents only some key causal processes while ignoring others; for accurate predictions concerning actual populations, the Lotka-Volterra model needs to be supplemented with additional causal factors.

This is one instance of how fine-graining the explanandum can change the causal nature of the associated explanation. Andersen (2016) identifies some other ways in which causal and non-causal explanations can complement each other, but all do so by targeting slightly different aspects of the same phenomenon.

It is interesting to note that fine-graining the explanantia can also change the causal nature of the associated explanation. For instance, Nehrlich (1979) considered the explanandum of a particle traveling along a curved line even though no force acts on it. The explanans lies in the geometry of space, and because, as Nehrlich argues, space is causally inert and there are no other causes of the particle's motion, the explanation is non-causal.

While many might not agree with Nerhlich's assessment of the example (for instance, in judging space to be causally inert he seems to assume a process rather than a difference-making account of causation), it is interesting to note how the causal nature of the associated explanation changes when the explanantia are finegrained. This is what seems to underlie the strategy Skow (2014: 451-2) takes in reassessing this example. He points out that relevant causal information remains hidden in Nehrlich's description of the explanans: if the dynamics governing particle motion were different, for instance by causing the particle to spontaneously prefer one direction over another, then the particle path would also be different. 
So there are other causes of the particle's motion beyond the geometry of space. Skow concludes that the resulting explanation is in fact causal.

Note that it would be inaccurate to state that Nehrlich and Skow disagree about the same explanation, since they are operating with different explanantia. In Nerhlich's analysis, the assumption is that particle dynamics cannot vary and hence does not feature in the explanans. In Skow's analysis, particle dynamics is a factor that can vary, and one on which the particle's movement counterfactually depends. In Skow's understanding of causal explanation (roughly, an explanation of an event $E$ is causal when it provides information about what could have prevented $E$ from occurring), this is sufficient to reassess the example as an instance of causal explanation.

Attention for granularity can also help make sense of the diverging intuitions in two otherwise similar accounts of the role of mathematics in scientific explanation: Strevens's (forthcoming) and Huneman's (2010, 2018). Huneman argues that mathematical properties non-causally explain by constraining the range of possible causal explanantia (e.g. causal histories, or causal mechanisms). This constraint thus explains a certain global property of the explanandum, one that counterfactually depends on the mathematical property. Strevens likewise holds that the explanatory function of mathematics is to constrain the set of possible difference makers, but reaches an opposite conclusion: far from constituting distinctively non-causal explanations, the use of mathematics serves only to reach a deeper causal explanation, where we not only identify the difference-makers, but also are able to explain why this and not some other factor is a difference-maker. There is no distinctively non-causal explanation, only different levels of causal 
explanation.

The divergence in assessment can be associated with an implicit difference in their description of the explanantia. To this end, consider the subtle differences in how "the seven bridges of Königsberg problem" is analyzed. In Strevens's account, causal explanation seeks to identify and isolate the causal differencemakers from the causal web consisting of all causal processes in the universe (Strevens 2008). Euler's explanation of the failure to cross all bridges without crossing the same bridge twice is, in this account, a highly abstract causal explanation: it is one that has abstracted away from all elements in the causal web that are not difference makers. The only difference-makers are the structure of the bridges and land masses (and the fact that walking around is equivalent to following a curve on a graph). Mathematics helps us identify the true differencemakers - it helps us understand why some elements are and why others are not causal difference makers - but it does not actually do the explaining. For Huneman, by contrast, the bridges example constitutes a non-causal explanation for the simple reason that the graph-theoretical property coarse-grains over all the different bridge configurations that instantiate that property. There are other bridge configurations that do not instantiate the theorem conditions. Even if the laws of nature were radically different, the counterfactual dependence of the outcome on the graph-theoretical property would hold. In this way, for Huneman, the graphtheoretical property coarse-grains the causal states; for Strevens, the same property identifies the relevant causal difference-makers.

The point here is not to confirm or criticize either stance, but rather point to a curious difference in the set of possible explanantia each philosopher works with. 
For Strevens, the explanans must be drawn from the causal web. The explanans that ultimately features in the causal explanation must be a causal differencemaker, but the set of 'candidate' explanantia (see next section) is the causal web. By contrast, the set of candidate explanantia for Huneman are alternative graphtheoretic theorems, only one of which is true in our world. The set of candidate explanantia in Strevens's analysis is thus much more fine-grained than in Huneman's analysis. Strictly speaking, they are thus not discussing the same explanation, since one operates with a different set of candidate explanantia than the other.

\section{GRANULARITY OF ANALYSIS}

In this section I will set out to make the preceding observations more precise. Not just explananda, but also explanantia can be fine-grained, and this seems to have at least something to do with diverging assessments of non-causal explanation. Later in the paper I will propose an explanation of this fact; by contrast, the purpose of this and next section is to offer a more precise definition of granularity, and of the distinction between causal and non-causal explanation.

Intuitively, fine-graining implies some sense of 'zooming in' on an object. A zebra and a horse may seem identical at a distance, but on closer inspection, we are able to perceive further properties, such as the patterns in their hides, which distinguish between the two animals. If we were to metaphorically 'zoom in' even further to the level of genotypes, we may distinguish between them as separate species. As an initial characterization of the concept, analyzing an object at a 'finer grain' can be understood to mean that the object is characterized by addi- 
tional properties, allowing a more powerful resolution by which the object can be distinguished from other types of object.

Similarly, we can 'zoom in' on a phenomenon. Consider a population of lynx and hare. As mentioned previously, an initial explanandum may be the simple fact that the relative frequencies of hare and lynx $(p, q)$ change periodically. This explanandum may be fine-grained to why the relative frequencies $(p, q)$ follow one particular sequence of numbers rather than another. Conversely, coarse-graining a phenomenon entails abstracting away from some properties, so that the resulting explanandum is indistinguishable from some other explananda within a wider class. Thus, instead of explaining why a lynx-hare population followed this particular path instead of another path towards an equilibrium $(p, q)$ (instead of equilibrium $\left(p^{\prime}, q^{\prime}\right)$ ), we may want to know why the equilibrium $(p, q)$ (instead of equilibrium $\left.\left(p^{\prime}, q^{\prime}\right)\right)$ was attained, regardless of path.

In general, an explanandum can be thought of as some proposition $p_{1}$ concerning the actual state of the world (e.g., a fact, the occurrence of an event, some state of affairs) that is contrasted with any number of other propositions $p_{2}, p_{3}, \ldots$ corresponding to alternative (but non-actualized) states of affairs. Let us assume for the sake of simplicity of exposition that $p_{1}$ concerns the instantiation of some property $P$ (" $X$ is $P$ "), and that the alternative proposition $p_{2}$ is the non-instantiation of that property (" $X$ is not- $P$ "), so that the explanandum is " $X$ is $P$ instead of not- $P$ ". This explanandum can be described at a finer grain by considering an additional property $Q$ which happens to be instantiated in $X$, and so the number of alternative propositions is increased to three, where either only $P$ is instantiated, or only $Q$, or neither of the two. 
Fine-graining the explanandum, as it will be understood in this paper, thus involves introducing contrast classes in specifying an explanandum (Van Fraassen 1980; Hitchcock 1996) 2 Fine-graining an explanandum involves introducing an additional contrast class, and monotonically increases the size of the set of propositions that could have been true but are not. If one is to scientifically explain a particular proposition about some part of the world, one has to explain why all the alternative propositions are not true.

A similar analysis can be done for fine- and coarse-graining explanantia, but a distinction must be made between two ways of fine-graining. Consider for instance the example of Euler's bridges. One set of initial states can could be the set of possible starting points a walker can take in Königsberg. This set of initial states can be fine-grained in two ways. The first is that we add further detail to the possible initial states: the walker starts out standing on one leg or on two legs, or starts out by crossing her fingers. This fine-graining allows factors to vary that had previously been ignored (without, of course, necessarily resulting in a better explanation.). The second way of fine-graining allows factors to vary that had previously been assumed to be constant: thus one can distinguish between initial states where graph theory (as we know it) is true, and initial states where an alternative graph theory is true (e.g., a graph theory, unimaginable to us but possible in some vastly extended sense of possibility, where all seven bridges can be crossed without crossing the same bridge twice).

To summarize this discussion, the level of granularity of an explanation can

\footnotetext{
${ }^{2}$ The inverse operation, coarse-graining, corresponds to introducing an equivalence class: see Desmond (2017).
} 
refer both to the granularity of the explanandum (how many alternative propositions there are with which the actually true proposition is to be contrasted) as well as to the granularity of the explanans (how many candidate propositions there are on which the explanandum depends). An explanandum is fine-grained by the introduction of a novel contrast class; an explanans is fine-grained by allowing a boundary condition to vary. Note that I do not assume any time-asymmetry for instance, in optimality explanations (discussed later more fully), possible explanantia can include different geometries or shapes which directly and without time-delay affect the possible equilibrium states of the system.

\section{A PRAGMATIC-MODAL ACCOUNT OF THE CAUSAL NATURE OF EXPLANATION \\ Modal Strength}

Since we want to account for and how granularity of description affects the boundary between causal and non-causal explanation, we need an initial representation of this boundary. In the following I will adopt Lange's use of the concept of modal strength $\mathrm{3}^{3}$ Lange's underlying intuition here is that, in non-causal explanations, the explanandum is entailed with a necessity that is not possible in causal explanations. For instance, regardless of what the laws of physics look like, it is impossible to cross all bridges of Königsberg without crossing the same bridge twice. No causal explanation could have entailed the explanandum with the same

\footnotetext{
${ }^{3}$ E.g., "If a fact has a distinctively mathematical explanation, then the modal strength of the connection between causes and effects is insufficient to account for that fact's inevitability." (Lange 2016: 6).
} 
'modal strength'.

In Lange's framework, degrees of modal strength are tied up with a metaphysics of necessities. The varieties of necessity form a pyramid-like structure, including accidents, natural necessities (such as natural laws), 'broadly logical' necessities (e.g. mathematical truths), and 'narrowly logical' necessity (logical truths, such as the law of noncontradiction). There is also a hierarchical relationship between these necessities. Natural necessity is 'lower' than mathematical necessity, in the sense that the number of counterfactual situations under which the laws are preserved is strictly less than the number of counterfactual situations under which the logical truths are preserved (see Lange 2009:77). Natural necessities hold true in a greater number of possible worlds than accidents do; logical necessities are true in an even greater number of possible worlds.

A distinctively mathematical explanation is then one that picks out one of these broadly or narrowly logical necessities on which an explanandum counterfactually depends. In contrast to Skow's conception of causal and non-causal explanation (cf. Lange 2016, 404 n.17), a distinctively mathematical explanation may implicitly involve causal dependencies, but derives its explanatory force from the logical necessity it picks out. For instance (cf. Lange 2016, 19ff), a mother may fail to distribute twenty-three strawberries evenly among her three children, and the number of strawberries and the number of children may be considered to be the causes of this failure: if the number of strawberries or of children were different, the she might have succeeded. However, the best explanation of the failure appeals to a mathematical fact, namely, that twenty-three is indivisible by three. The numbers of strawberries and children are only explanatorily relevant 
insofar as they allow for this mathematical fact to be applied. It is the mathematical fact that grounds the non-causal nature of the explanation, and that allows the explanandum to be entailed across all possible configurations of the causal web - even regardless of whether a possible world is populated with a web of pseudo-processes instead of causal processes (cf. Salmon 1984).

In the following I will bracket Lange's metaphysics, and consider modal strength purely from a pragmatic point of view. So the approach is non-ontic in the sense that the question of what parts of an ontology ground different degrees of modal strength is bracketed. Instead, modal strength is considered purely as a property of the internal structure of explanation, and this is connected to how explanations are used and their causal character judged. The following is a qualitative statement of the pragmatic conception of modal strength:

An explanation is assessed as non-causal if the explanandum's degree of necessity is independent of explanans; and otherwise it is deemed causal.

At this point, this statement is simply stipulated; the motivation and argument for its plausibility will be given in due course. For the moment it is sufficient to note the pragmatic and non-ontic use of modal strength: judging an explanation to be causal or not depends entirely on how the explanandum and explanans are represented within an explanation, not on whether the explanation is true or picks out genuine causal/non-causal dependencies.

In the following subsection I use some generic features of the interventionist approach to explanation in order to develop this conception of modal strength 
with some more quantitative precision. This helps make sense of how precisely the choice of explanandum/explanans affects the degree of modal strength.

\section{A quantitative measure}

A powerful way to analyze explanations quantitatively is through the interventionist framework, where the explanandum and explanans are conceived as variables $\stackrel{4}{4}^{4}$ In this subsection I will adopt this approach, and will formalize explanans and explanandum as variables, but I will divorce this from conceiving of the relation between explanans and explanandum in terms of physical interventions. Instead the relation will be conceived of as a conditional probability, to allow for noncausal explanations to be represented within the framework as well.

In particular, let the triple $(Y, X, R)$ represent the structure of an explanation, where $Y$ is the explanandum variable, $X$ is the explanans variable, and $R$ the set of conditional probabilities $\left\{P\left(y_{k} \mid x_{j}\right) \mid x_{j} \in X, y_{k} \in Y\right\}$. It is useful to allow $X$ and $Y$ to also denote the sets of possible values the explanandum and explanans variables can take. The explanandum consists of the set $Y$, consisting of a particular value $\{y\} \in Y$ together with its contrast class $Y \backslash\{y\}$. The explanans consists of $X$ and $R$. The set $X$ is defined by the range of possible values, and thus implicitly reflects the boundary conditions: certain conditions that all explanans values meet ${ }^{5}$ The

\footnotetext{
${ }^{4}$ From Woodward and Hitchcock 2003, 6: “The explanandum is a true (or approximately true) proposition to the effect that some variable (the 'explanandum variable') takes on some particular value. The explanans is a set of propositions, some of which specify the actual (approximate) values of variables (explanans variables), and others which specify relationships between the explanans and explanandum variables.” See also Hitchcock 1996.

${ }^{5}$ This is why mathematical facts can play a role in explanations even though they are not ex-
} 
relation $R$ represents how $X$ maps onto $Y$.

This may not be the only way to quantitatively represent explanations; however, it assumes only a very basic way of representing explanatory structure that most if not all accounts of explanation share, including traditional accounts such as deductive-nomological explanation and statistical-relevance explanation, as well as more recent ones such as the kairetic account (Strevens 2008) or the counterfactual theory of explanation (Reutlinger 2016). Of course, given this level of generality, it should come as no surprise that triples $(Y, X, R)$ do not actually distinguish between explanatory and non-explanatory relations between explanans variable and explanandum variable (accounts of explanation typically provide extra conditions on the relation, such as invariant generalizations, or law-like connection). However, the only purpose here is to elucidate the relation between grain of analysis and causal nature.

Given these preliminaries, the two following notions of modal strength can be considered:

(1) An explanandum value $(Y=y)$ is entailed with non-causal modal strength within explanation $(Y, X, R)$ if the probability $P(Y=y \mid X=x)$ is one or zero for every $x$.

(2) An explanandum value $(Y=y)$ is entailed with non-causal modal strength within explanation $(Y, X, R)$ if the probability $P(Y=y \mid X=x)$ is equal for all $x$.

plicitly represented by the explanans variable: mathematical facts can act as boundary conditions (see later). 
Definition (1) captures how some outcome is inevitable (or impossible) within the context of a particular explanation $(Y, X, R)$. Definition (2) captures how the occurrence of some explanandum value is independent of the explanans variable.

However, definition (1) is a flawed way of capturing the notion of 'modal strength'. For example, consider the explanation of the question, "why did Paul hit the bull's eye of a dart board given that Paul threw the dart at a certain velocity $\mathbf{v}$ ?" If $X$ is defined as the set of all possible velocities, and $Y$ the set of all possible end points of the dart after it is thrown, the probability of the outcome state "bull's eye", given a relatively narrow range of velocities $\tilde{x} \subset X$ is one (by the equations of kinetics, and some boundary conditions like no sudden gusts of wind). Outside this narrow range, the conditional probability of a bull's eye is zero $(P(y \in \tilde{y} \mid x \in$ $X \backslash \tilde{x})=0$ ). However, this is clearly a causal explanation: if Paul had thrown the dart with a different velocity, it might not have hit the bull's eye. The initial velocity of the dart - a causal factor that the explanation assumes could have been different in different arrangements of the causal network of the universe - is counterfactually relevant for the explanandum.

How would $(Y, X, R)$ have to be redefined so that the explanation would have a non-causal character? Consider how the explanation changes when the question changes to "why did Paul hit the bull's eye when throwing at some velocity (regardless of whether he used a catapult, his arm, or a gun to give the dart the initial velocity)?" In this case, the set of explanans values is constrained to $\tilde{x}-$ the initial velocity is now a boundary condition instead of an explanans variable. Instead, explanans values are distinguished by the cause of the initial velocity. The best explanation of this new question will refer to the properties of kinematics, and more 
specifically to certain geometrical properties entailed by vector calculus. Hence the resulting explanation is non-causal.

As a second example, consider a case where the explanandum is not implied with probability one or zero. For instance, consider the explanation of why Schrödinger's cat died. Schrödinger put his cat in a box together with a decaying uranium atom, a Geiger counter, and some poisonous gas which would be released when the Geiger counter registered the decay of the atom. Now, according to quantum theory, after some time has passed (the half-life of uranium in this case), the two possible outcomes (cat is alive or cat is dead) have probability $1 / 2$. Then I open the box and see that the cat is alive. What explains this outcome? The explanation is causal if, in the definition of the set of initial states $X$, I allow the fundamental properties of uranium to vary; the explanation is non-causal if I hold such properties to be fixed, and only allow other possible initial states to vary, such as whether I open the box gently or abruptly, or whether the box is made out of cardboard or iron.

What these two cases show is that the size of the probability of the explanandum within the context of an explanation does not matter for whether the explanation is causal or not. What matters is whether the entailment relation is affected by the explanans values that are allowed to vary. What matters is not whether the explanandum is shown to be inevitable, but rather that the entailment of the explanandum is shown to be independent of explanans values.

Given this motivation for definition (2), one can propose the following informationtheoretic measure as an operationalization of modal strength:

Within an explanation $(Y, X, R)$, the explanandum value $y$ (instead of 
its contrast class $Y \backslash\{y\}$ ) is entailed with non-causal modal strength if and only if the mutual information $I(X ; Y)$ equals zero.

Mutual information $I(X ; Y)$ is defined as $H(Y)-H(Y \mid X)$, the difference between the unconditional entropy over outcome states and the conditional entropy over outcome states. The entropy $H$ of a probability distribution (conditional or unconditional) is a measure of the uncertainty involved in predicting what the precise outcome will be. If the probability distribution is uniform, the entropy is maximal; if one outcome has probability one, the entropy is zero. Mutual information, by contrast, does not measure uncertainty, but rather 'uncertainty reduction' given knowledge of an explanans value $x$. If knowing $x$ affects the predictability of a possible outcome $y$, then this knowledge reduces the uncertainty of the outcome. Thinking of mutual information in terms of uncertainty reduction is sufficient for our purposes here, but for precise definitions, see Cover and Thomas (2006) or Author (2017).

This definition of the degree of modal strength captures key properties of modal strength as defined in (2). First, mutual information is zero when knowing that one explanans value rather than another occurred does not affect the probability of the explanandum variable taking a particular value. The unconditional probability of the outcome - which assumes that any initial state could occur - is the same as the conditional probability of the outcome, where there is knowledge that only one initial state occurs.

Definition (2) of modal strength is the core of the pragmatic-modal account of the causal nature of explanation. It is not an account of causal explanation, because it makes no attempt to pinpoint how structures such as $(Y, X, R)$ are ex- 
planatory in the first place. Thus, it is not a competitor with the ontic view on causal explanation. The target of the pragmatic-modal account is rather to identify the conditions under which we will judge a given explanation to be causal, or to be non-causal.

In this sense, it can also be contrasted with the 'modal view of explanation' (e.g. Mellor 1976, see discussion in Salmon 1989, 118ff), which does aim to be a full competitor of the ontic view. According to the modal view, explaining some event or phenomenon means showing that it happened with necessity given the explanans (universal laws and particular conditions). Mellor's version of the modal view is slightly weaker, requiring only that explanation show that the explanandum was necessitated to a certain degree ('partial entailment'). Degree of necessitation is identified with the size of the conditional probability of the explanandum given the explanans. By contrast, in the pragmatic-modal account, no account of explanatoriness is given, and the size of the probability does not matter for judging an explanation to be causal or not.

\section{APPLICATION}

Until now the reader has been motivated to go along with the pragmatic (nonontic) analysis of modal strength, as well as (to a lesser extent) with the quantitative operationalization of modal strength. We will now consider some harder arguments for the virtues of the pragmatic-modal account. I will do so by outlining three areas where the account is philosophically fruitful: accounting for the fundamental connection between granularity and the causal nature of explanation; dissolving disagreements concerning the causal nature of specific examples; 
and dissolving (at least some) disagreements concerning the definition of causal explanation.

\section{Modal Strength and Granularity}

Recall that in the ontic view of causal (as well as of non-causal) explanation, it is somewhat of a mystery why fine-graining an explanans should not reveal anything but causal explanations, or anything but non-causal explanations. If a vaguely described explanation is like a grey-scale photograph, why should zooming in reveal darker and brighter shades, and not just the same shade of grey? By contrast, within the pragmatic-modal framework, a natural connection can be made between granularity and modal strength.

First let's consider an intuitive description of this connection. To recapitulate from the previous section, an explanandum variable is explained non-causally when the probabilities of the occurrence of the explanandum values are not affected by the explanans variable. Hence, if the explanandum is fine-grained, there are more explanandum values where there could be a dependence on the explanans variable. Thus it will be 'more difficult' (as it were) to show that the various 'explanans values' no longer matter. As an example, consider the use of the Lotka Volterra model to explain why a particular limit cycle $\Lambda$ was reached (within the context of a Lotka-Volterra explanation). The set of initial states is the set of initial relative frequencies; the set of possible outcomes is the set of possible limit cycles. Knowing the precise initial state is not relevant for what limit cycle will occur; the

limit cycle $\Lambda$ can be deduced as a mathematical consequence of certain structural features of the equation - the explanation is thus non-causal. However, if the set 
of outcome states is fine-grained to consist not of possible limit cycles, but of possible frequencies sequences followed by a population over time $t,\left(p_{t}, q_{t}\right)$, there will be many possible paths corresponding to a single limit cycle (but not vice versa). Here knowing the precise initial frequencies $\left(p_{0}, q_{0}\right)$ will be relevant for the resulting outcome: the explanation is causal.

However, not every fine-graining of the explanandum of a non-causal explanation yields a causal explanation. The failure to cross the seven bridges of Königsberg in the right way is not affected by the starting position, but a finegraining of this explanandum - for instance, the failure to cross the bridges in a particular order - is not affected by the starting position either. This makes sense: the outcome of crossing all seven bridges in the right way is impossible given the set of possible initial states, and this means that every particular way of crossing the seven bridges is impossible too.

This intuition is encapsulated by the following theorem:

Fine-graining the set of explanandum values (or explanans values) monotonically increases the causal character of the explanation.

Proof. Let $Y=\left\{y_{1}, \ldots, y_{n}\right\}$ be the set of outcome states, and consider the finegraining $Y^{\prime}$ where $y_{n}$ is replaced by $\left\{w_{1}, \ldots, w_{m}\right\}$, so that $Y^{\prime}=\left\{y_{1}, y_{2}, \ldots, y_{n-1}, w_{1}\right.$, $\left.\ldots, w_{m}\right\}$.

Then, relying on the log sum inequality (cf. Cover and Thomas 2006, 31):

$$
\begin{aligned}
I\left(Y^{\prime} ; X\right) & =\sum_{y^{\prime}} \sum_{x} P\left(y^{\prime}, x\right) \log \left(\frac{P\left(y^{\prime}, x\right)}{P\left(y^{\prime}\right) P(x)}\right) \\
& =\left[\sum_{y^{\prime}=w_{1}}^{w_{m}} \sum_{x} P\left(y^{\prime}, x\right) \log \left(\frac{P\left(y^{\prime}, x\right)}{P\left(y^{\prime}\right) P(x)}\right)-P\left(y_{n}, x\right) \log \left(\frac{P\left(y_{n}, x\right)}{P\left(y_{n}\right) P(x)}\right)\right]+I(Y ; X) \\
& \geq I(Y ; X)
\end{aligned}
$$


Because mutual information is symmetric $(I(X ; Y)=I(Y ; X))$, the same proof is applicable to fine-graining the explanans values. Furthermore, a sufficient condition to reach equality is when $P\left(y_{n}, x\right)=0$ for all $x$, i.e., when the explanandum value $y_{n}$ is impossible, regardless of the value of the explanans variable.

Note that this theorem is a limited result. What it does not say is that the associated explanation of a fine-grained explanandum has a more causal character. Rather, this result only holds if the explanans is held constant throughout the fine-graining operation. If the fine-grained explanandum necessitates a different explanans variable, then the associated explanation may have a very different modal structure, anywhere between the causal or non-causal end of the spectrum.

Nonetheless, even this limited result can help give deeper insight into why fine-graining an explanandum should affect the causal nature of the associated explanation. It also identifies two previously overlooked properties. The first is the importance of fine-graining an explanans variable: this is discussed in more detail in the next section. The second is that some non-causal explanations retain their non-causal character no matter how much the explanandum is fine-grained (when the equality condition in the proof above is satisfied: e.g, the bridges of Königsberg example). Likewise, the causal character of an explanation can reach a maximum when knowledge of the explanans value allows the explanandum value to be specified with absolute certainty (i.e., the case of determinism). In this case, fine-graining the explanandum will not further alter the causal character of the explanation. To return back to the metaphor of the photograph: some grains remain black and some grains remain white, no matter how much you zoom in on 
them.

\section{Analyzing Diverging Assessments I}

\section{Constraint explanations}

We can now revisit the contrast between Strevens's and Huneman's diverging assessments of the role of mathematics in scientific explanation. For Strevens, an explanans variable can possibly range over any aspect of the causal web. The explanatory role of mathematics is to help us abstract away from those aspects of the causal web that do not make a difference for the explanandum variable, and thus to help us identify the true causal difference-makers. The mathematical proposition thus is a condition for what aspects of the causal web can qualify as a true causal difference-maker, but the mathematical proposition does not itself represent a value the explanans variable can assume. In any case, for Strevens, in a given constraint explanation the explanans variable ranges over any difference-making aspect of the causal web, so that knowing what value the explanans variable takes affects the probability of what value the explanandum variable will take. In other words, the explanation is causal.

By contrast, for Huneman - at least according a basic construal of his account - the mathematical property or proposition acts as a boundary condition on the explanans variable. The explanans variable ranges only over configurations or states of affairs that instantiate a certain mathematical property, or that allow a certain mathematical theorem to be applied. (So Huneman's explanans variable ranges over fine-grained states of affairs that instantiate a relevant topology, whereas Strevens's explanans variable ranges over coarse-grained states of affairs 
that both do and do not instantiate the relevant topology.) In other words, the explanans variable thus makes no difference for the probability of the explanandum variable. The explanation is non-causal.

Something similar is implicitly going on in Lange's analysis of the strawberry example. While Lange acknowledges that the number of strawberries and the number of children are causal difference makers for the explanandum value (i.e., failure to distribute strawberries evenly), and thus can be considered different values of the explanans variable, the set of explanans values is coarse-grained according to whether a mathematical property is instantiated or not (i.e. one number being indivisible by another). When emphasizing the 'distinctively mathematical' aspect of this explanation, Lange is implicitly speaking of the mathematical property as a boundary condition.

It is possible to identify a third construal that combines parts of the previous two. Allow the explanans variable to range over all possible configurations or states of the physical system under consideration. Only some of these configurations instantiate the mathematical property (topological, structural, etc.) that is being invoked as explanatory. So here the explanans variable ranges over finegrained states of affairs that both do and do not instantiate the relevant topology. Strevens's construal is obtained by coarse-graining the explanans variable: the mathematical property induces an equivalence class on the values of the explanans variable, such that two explanans values are considered equivalent if they correspond to instantiations of the same mathematical property. In this explanation, knowing the value of the explanans variable only matters insofar knowing which equivalence class the value is as part of. 
Note that the third construal is a fine-graining of (my reading of) of Strevens's construal, but that this fine-graining does not increase the causal character. By contrast, the third construal is also a fine-graining of (my reading of) Huneman's construal but does increase the causal character. This can be seen as follows: in the third construal the relevant mathematical property is allowed to be variably instantiated, but in Huneman's construal the mathematical property is taken as a boundary condition. (This is a coarse-graining because the explanans values are characterized by fewer properties.) This is an illustration of how not all fine-grainings have the same effect on the causal character of the associated explanation.

\section{Optimization explanations}

Consider optimization phenomena as a second example of how mutual information may help analyze controversial examples of scientific explanation. The canonical example here is the the marble finding its way to the bottom of the bowl, regardless of where on the bowl's rim it is initially placed (see Sober 1983). Sober argued (a view echoed more recently in Rice 2015) that optimization explanations are not causal because they do not cite the precise path followed by the marble - instead, the outcome state is explained as an attractor state given certain structural features, such as the geometry of the bowl. This would be a construal of the explanation where the explanans variable ranges over possible paths a marble may take, and structural features function as a boundary condition.

However, it is possible to give optimization explanations a causal construal, in analogy with constraint explanations. Strevens has argued $(2008,271)$ that such explanations are causal because they pick out difference-makers: not just the 
geometry of the bowl, but also the fact that the marble is placed somewhere on the bowl's rim. The explanans variable range over all difference-making aspects of the causal web, including possible geometries of the bowl, the smoothness of the bowl surface, as well as all the possible starting positions of the marble (including a starting point outside the bowl). Only some of these values will lead to the outcome where the marble finds its way to the middle (and bottom) of the bowl. This is a causal explanation.

\section{Analyzing Diverging Assessments II}

In light of the preceding discussion, a tentative suggestion can be made that, to some extent, what seem like disagreements about the definition of causal explanation are to some extent really disagreements about what explanans variable to use. Ostensibly, there seems to be a divergence in the definition of causal explanation between, say, Lange and Skow, and at first sight this divergence may seem to explain why different assessments are reached about particular explanations. However, upon closer analysis, it could be argued that Lange and Skow operate with different explanans variables. Skow's preferred definition of causal explanation is, in his own formulation, an explanation that cites "what causes, if any, [the explanandum] had; or if it [cites] what it would have taken for some specific alternative or range of alternatives to [explanandum] to have occurred instead." (Skow 2014, 449). So, an explanation is causal if either the explanans variable ranges over the causal difference-makers; or, if there seems to be no identifiable cause of the a phenomenon, and the explanans variable ranges over causal differencemakers of the other possible (but non-actualized) values of the explanandum vari- 
able. In either case, knowing what value the explanans variable assumes reduces the uncertainty over what value the explanandum variable will obtain.

By contrast, and as previously analyzed, for Lange the explanans variable is either coarse-grained according to whether a mathematical property is instantiated or not, or the mathematical property simply acts as a boundary condition on the range of values of the explanans variable. The latter case describes 'distinctively mathematical' explanations, and the explanans variable is allowed to roam over all possible worlds where the laws of nature might look very different - just as long as the mathematical property is instantiated.

The contrast between Strevens's and Huneman's definition of causal explanation is perhaps less dramatic, but nonetheless Strevens operates with his own (kairetic) account of difference-making where difference-makers are abstracted from the causal web, whereas Huneman seems to operate with a mechanistic conception of causation (at least in Huneman 2018). Here too, the divergence is less deep that it would seem: as previously argued, each gives a different construal of constraint explanations, with different explanans variables.

\section{CONCLUSION}

The ontic view of the causal nature of explanation is: causal explanations pick out causal dependencies between explanans and explanandum, and non-causal explanations pick out non-causal dependencies. Confusion as to the causal nature of instances of explanation arises when the explanation is not precisely described. The pragmatic-modal account is not a full competitor of the ontic view - it does not attempt to give an account of explanation as such in the way that e.g., Reutlinger 
2016 does - but it does compete with it in giving an account of why fine-graining the explanandum and explanans seems to affect our assessment of the causal nature of the resulting explanation. I have argued that the pragmatic-modal account is superior because, unlike the ontic view, it does not write off the connection between granularity and causal nature as a coincidence. Rather, the connection reflects basic and generic features of the structure of explanation. Furthermore, I have suggested how the pragmatic-modal account can help dissolve not only diverging assessments of explanations, but even diverging accounts of causal explanation. Disagreements about causal and non-causal explanation perhaps do not go quite as deep as sometimes assumed, and can be avoided by greater precision in defining not just explanandum variables but also explanans variables. 


\section{REFERENCES}

Andersen, Holly. 2016. "Complements, Not Competitors: Causal and Mathematical Explanations." The British Journal for the Philosophy of Science, August, axw023.

Cover, Thomas M., and Joy A. Thomas. 2006. Elements of Information Theory. Wiley Series in Telecommunications. New York, USA: John Wiley \& Sons, Inc. Craver, Carl F. 2014. "The Ontic Account of Scientific Explanation.” Pp. 27-52 in Explanation in the Special Sciences. Edited by Marie I. Kaiser, Oliver R. Scholz, Daniel Plenge, and Andreas Hüttemann. Dordrecht: Springer Netherlands.

Desmond, Hugh. 2017. "Symmetry Breaking and the Emergence of PathDependence." Synthese 194 (10): 4101-4131.

Hitchcock, Christopher Read. 1996. "The Role of Contrast in Causal and Explanatory Claims.” Synthese 107 (3): 395-419.

Huneman, Philippe. 2010. 'Topological Explanations and Robustness in Biological Sciences.” Synthese 177 (2): 213-45.

—. 2018. "Outlines of a Theory of Structural Explanations." Philosophical Studies 175 (3): 665-702.

Lange, Marc. 2009. Laws and Lawmakers: Science, Metaphysics, and the Laws of Nature. Oxford, UK: Oxford University Press. 
—. 2017. Because without Cause: Non-Causal Explanation in Science and Mathematics. Oxford Studies in Philosophy of Science. New York, NY: Oxford University Press.

Mellor, David H. 1976. "Probable Explanation.” Australasian Journal of Philosophy 54 (3): 231-41.

Nerlich, Graham. 1979. "What Can Geometry Explain?" The British Journal for the Philosophy of Science 30 (1): 69-83.

Reutlinger, Alexander. 2016. "Is There a Monist Theory of Causal and Noncausal Explanations? The Counterfactual Theory of Scientific Explanation.” Philosophy of Science 83 (5): 733-745.

Rice, Collin. 2015. "Moving Beyond Causes: Optimality Models and Scientific Explanation.” Noûs 49 (3): 589-615.

Salmon, Wesley C. 1984. Scientific Explanation and the Causal Structure of the World. Princeton University Press.

Skow, Bradford. 2014. "Are There Non-Causal Explanations (of Particular Events)?" The British Journal for the Philosophy of Science 65 (3): 445-67.

Sober, Elliott. 1983. "Equilibrium Explanation.” Philosophical Studies 43: 20110.

Strevens, Michael. 2008. Depth: An Account of Scientific Explanation. Cambridge, MA: Harvard University Press.

—. (forthcoming). "The Mathematical Route to Causal Understanding." In Explanation Beyond Causation. Edited by Alexander Reutlinger and Juha Saatsi. Oxford: Oxford University Press. 
Van Fraassen, Bas C. 1980. The Scientific Image. Oxford, UK: Oxford University Press.

Woodward, James. 2003. Making Things Happen: A Theory of Causal Explanation. Oxford, UK: Oxford University Press.

Woodward, James, and Christopher Hitchcock. 2003. ”Explanatory Generalizations, Part I: A Counterfactual Account.” Noûs 37 (1): 1-24. 